\title{
Long period variables detected by ISO in the Small Magellanic Cloud ${ }^{\star}$
}

\author{
M.-R. L. Cioni ${ }^{1}$, J. A. D. L. Blommaert ${ }^{2}$, M. A. T. Groenewegen ${ }^{2}$, H. J. Habing ${ }^{3}$, J. Hron ${ }^{4}$, F. Kerschbaum ${ }^{4}$, \\ C. Loup ${ }^{5}$, A. Omont ${ }^{5}$, J. Th. van Loon ${ }^{6}$, P. A. Whitelock ${ }^{7}$, and A. A. Zijlstra ${ }^{8}$ \\ ${ }^{1}$ European Southern Observatory, Karl-Schwarzschild-Str. 2, 85748 Garching bei München, Germany \\ 2 Institute of Astronomy, Katholieke Universiteit Leuven, Celestijnenlaan 200B, 3001 Leuven, Belgium \\ 3 Sterrewacht Leiden, Niels Bohrweg 2, 2333 RA Leiden, The Netherlands \\ ${ }^{4}$ Institut für Astronomie der Universität Wien, Türkenschanzstrasse 17, 1180 Wien, Austria \\ 5 Institute d'Astrophysique de Paris, CNRS, 98bis Bld. Arago, 75014 Paris, France \\ 6 Astrophysics Group, School of Chemistry and Physics, Keele University, Staffordshire ST5 5BG, UK \\ 7 South African Astronomical Observatory, PO Box 9, 7935 Observatory, Republic of South Africa \\ 8 Department of Physics, UMIST, PO Box 88, Manchester M60 1QD, UK
}

Received 8 January 2003/ Accepted 18 April 2003

\begin{abstract}
This article presents the study of the light-curves extracted from the MACHO database of a sample of stars observed by the Infrared Space Observatory in the Small Magellanic Cloud. These stars belong to the ISO-Mini-Survey catalogue of the Magellanic Clouds (ISO-MCMS, Loup et al. in preparation). Most of them are in the asymptotic giant branch (AGB) and supergiant phases. The dominant period and amplitude of pulsation have been derived and the stars have been classified as Mira or Semi-Regular pulsators. Furthermore, the cross-identification with near-infrared DENIS and 2MASS magnitudes available within the ISO-MCMS allowed us: (i) to investigate the properties of these stars in the combined near- and mid-infrared colour-magnitude diagrams, (ii) to derive the bolometric magnitude by integrating the spectral energy distribution and (iii) to estimate the mass-loss rate. The stars have been divided into carbon- $(\mathrm{C}-)$ and oxygen-rich $(\mathrm{O}-\mathrm{rich})$ using the $\left(J-K_{S}, K_{S}\right)$ colour-magnitude diagram and their period and amplitude distributions have been compared. C-rich AGB stars have a sharp peak in their period distribution at about 250 days and have on average a larger amplitude than O-rich AGB stars. This effect, not previously detected from the study of similar stars in the Large Magellanic Cloud and in the Baade's window, might be closely related to the metallicity of the environment in which the stars have formed.
\end{abstract}

Key words. galaxies: Magellanic Clouds - infrared: galaxies - stars: mass loss - stars: variables: general

\section{Introduction}

The stellar content of the Small Magellanic Cloud (SMC) can be studied in great detail because of the low galactic extinction and known distance $m-M=18.99 \pm 0.03$ ( formal $) \pm$ 0.08(systematic), Cioni et al. (2000). However, it is well known, but not yet quantified, that the SMC is extended along the line of sight much more than its companion galaxy, the Large Magellanic Cloud (LMC). These two galaxies and the Milky Way galaxy constitute an interacting system (e.g. Gardiner \& Noguchi 1996; Moore \& Davis 1994; Heller \& Rohlfs 1994) and a metallicity scale environment in which the Galaxy is the most metal rich and the SMC the most metal poor of the three. Fields covering almost the whole of the two Magellanic Clouds and a considerable number of fields

Send offprint requests to: M.-R. L. Cioni, e-mail: mrcioni@eso.org

* Complete Tables 1 and 4 are only available in electronic form at the CDS via anonymous ftp to cdsarc.u-strasbg. fr

(130.79.128.5) or via

http://cdsweb.u-strasbg.fr/cgi-bin/qcat?J/A+A/406/51 in our Galaxy have been repeatedly observed by the microlensing projects: OGLE, MACHO and EROS. This provides a wealth of data to identify and study variable stars in each evolutionary phase. Partial or all-sky survey data in the nearinfrared (IR) provide essential counterparts for the specific analysis of the reddest objects: red (RGB) and asymptotic giant branch (AGB) stars. Most AGB stars are pulsating with an amplitude of up to a few magnitudes in the optical wave bands and somewhat less in the near-infrared bands. Long Period Variables (LPVs) have a period of at least 100 or 150 days and longer. Many are also multi-periodic objects. More than one dependence between period and luminosity has been detected in the LMC (Wood et al. 1999; Cioni et al. 2001; Noda et al. 2002; Lebzelter et al. 2002). Each period-luminosity relation (PLR) is probably associated with a different mode of stellar pulsation. Because most of these stars are probably experiencing the thermal pulsing AGB phase (TP-AGB), they are losing mass at an average rate of $10^{-6} M_{\odot} \mathrm{yr}^{-1}$ and they have highly extended atmospheres. The pulsation as the driving 
mechanism of the stellar wind is a fundamental element in the study of the mass-loss rate of AGB stars. They are surrounded by an initially optically thin, and later optically thick, circumstellar envelope and thus sometimes become detectable only in the infrared wave bands. Many obscured AGB stars were discovered by the IRAS satellite. More recently the Midcourse Space Experiment (MSX - Price et al. 2001) observed selected high density regions in the Magellanic Clouds. Improved source positions were derived. Egan et al. (2001) present the cross-identification between MSX and 2MASS data in the LMC. However, the Infrared Space Observatory (ISO) obtained more sensitive data in some fields in the Magellanic Clouds and the Galaxy that increased the number of obscured AGB stars and allowed us to learn more about their dust properties.

In order to study the evolved AGB population it is thus important to utilize observations that cover the spectral range from the optical ( $I$ band) to the mid-IR. It is then possible to derive fundamental quantities such as the bolometric magnitude and the mass-loss rate of the stars. In addition, from the period, the amplitude and the regularity of the variation, it is possible to characterise the stars as Mira or Semi-Regular (SR) pulsators and to indicate, from the PLR, the mass of the stellar progenitor. In this article the properties of a sample of AGB stars in the SMC extracted from the ISO-Mini-Survey catalogue of the Magellanic Clouds (ISO-MCMS, Loup et al. in preparation), which already contains the cross-identification with the near-IR catalogues DENIS and 2MASS, are discussed. This is done by additionally analysing the light-curves extracted from the MACHO database. A similar work was performed in the LMC bar-west field (Cioni et al. 2001) and will be further extended to other fields where ISO measurements are now available. A detailed comparison of more or less obscured AGB variables between the two Clouds and the Baade's window NGC 6522 will be given in another paper (Glass et al. in preparation).

The data and the cross-identification with the light-curves are described in Sect. 2. In Sect. 3 the analysis of the period and amplitude of variability and the determination of the bolometric magnitude is presented. A discussion of the different type and variability class of the sources in the PLRs and in the near- and mid-IR colour-magnitude diagrams is given in Sect. 4. Section 5 concludes the article. Appendix A discusses the re-analysis of the LPVs studied by Feast et al. (1989) and $\mathrm{B}$ compares the bolometric correction obtained in this work with the relations by Alvarez et al. (2000) and Montegriffo et al. (1998).

\section{Data}

The sample of data in the SMC, analysed here, is based on the cross-identification between the ISO-MCMS (Loup et al. in preparation), and the MACHO light-curves publicly available $^{1}$.

The ISO mini-survey in the direction of the SMC covers an area of 0.28 square degrees. Observations were performed with the infrared camera ISOCAM on board the ISO

\footnotetext{
1 http://wwwmacho.mcmaster.ca
}

satellite in the $L W 2(6.61 \mu \mathrm{m})$ and $L W 10(11.18 \mu \mathrm{m})$ wavebands. The catalogue lists 1333 sources brighter than about 12.4 and 11.3 mag in $L W 2$ and $L W 10$, respectively. The coordinates of the ISO sources are better than 4 " with a faint tail up to $6 "$. The catalogue also lists the cross-identification with the near-infrared catalogues 2MASS (2nd Incremental Release PSC $^{2}$ ) and the DENIS (Epchtein et al. 1997) catalogue towards the Magellanic Clouds (DCMC - Cioni et al. 2000a), and a few smaller optical catalogues of carbon stars, HII regions, PNe and emission-line stars. According to the association likelihood criteria defined by Loup et al. (in preparation) $48 \%$ of the sources have no, or an unlikely, association with DENIS/2MASS; $13 \%$ have a questionable association; $25 \%$ have a confident association and the remaining $14 \%$ are "known" sources with a DENIS/2MASS and optical counterpart. It is perhaps surprising that there are many ISO sources without a near-infrared counterpart. However, most of these sources are among the faintest ISO detections in both ISO wavebands. The histogram of their magnitudes and colour peaks at: $L W 2=12, L W 10=11$ and $(L W 2-L W 10)=1.5$. Moreover, the ISO-MCMS includes sources well detected in one band only. Among sources detected only in two bands about $86 \%$ have a near-IR counterpart. The ISO photometric magnitudes are part of the final version of the catalogue released to the Co-Is and associates in March 2002.

The MACHO (Alcock et al. 2000) light-curves were obtained from repeated measurements in two, blue and red, broad pass-bands from the 50 inch telescope at the Mount Stromlo Observatory in Australia. Observations of fields covering most of the Magellanic Clouds were performed from 1992 to 2000. The light-curves are easily accessible via the web interface giving in input the right ascension (RA) and the declination (DEC) of each source.

Figure 1 shows the regions observed by ISO superimposed on the MACHO fields. The area covered by ISO is entirely contained in MACHO fields number 211 and 212; a few sources are also present in field number 207. These MACHO fields were observed for the whole period of eight years while the observations of fields in the external parts of the SMC cover just five years. The OGLE light-curves cover a period of about five years (observations started in 1997). Because of the nature of LPVs, sources with periods from 100 to 1000 days, the MACHO light-curve database was preferred.

\subsection{Match between ISO-MCMS and MACHO}

Each ISO source with a confident DENIS/2MASS association was searched for a light-curve in the MACHO database using, as input, the 2MASS coordinates. Variables were confidently identified within a radius of $1^{\prime}$. Fig. 2 shows that most of the sources were actually associated within $1^{\prime \prime}$. In a very few cases the MACHO counterpart, a source with a typical LPV light-curve, was not the closest to the 2MASS source - but still within no more than $4^{\prime \prime}$. We consider a light-curve to be that of a LPV when a more or less periodic behaviour is detected by eye. It is expected that most of the sources detected

\footnotetext{
${ }^{2}$ http://www.ipac.caltech.edu/2mass/
} 
in the near- and mid-IR are associated with AGB stars. This is a consequence of the mass-loss process occurring in AGB stars that builds up a circumstellar envelope observable in the midinfrared.

Among the 502 sources with a confident DENIS/2MASS counterpart, 458 have been found to have a MACHO lightcurve. Table 1, available electronically at the CDS, lists the MACHO coordinates at the epoch J2000 (Cols. 1 and 2), the MACHO identifier field, tile and number (Cols. 3-5), the distance in arcsec between the 2MASS and the MACHO source (Col. 6) and the ISO-MCMS identifier (Col. 7). The first 10 lines are given as an example (Table 1).

The sources listed in Table 2 are those for which we could not find a convincing MACHO counterpart. The ISO-MCMS name is given in Col. 1, the distance in arcsec to the first possible MACHO counterpart is given in Col. 2 and in some cases the reason for the missing cross-identification is written in Col. 3. Too few data points means that there are not enough points to characterise the shape of the light-curve. This would be useful to recognize a MACHO counterpart within the ISO astrometric precision.

\section{Period analysis}

The Schwarzenberg-Czerny method (1996) has been used to detect periodicities in the light-curves of the selected sources, independently for the measurements in the blue and red bandwidth of MACHO. The algorithm was executed thirty times searching for periods in a range from 10 to $100,200, \ldots$, 3000 days with a step of 0.005 days. The result of the program is the period of the variation and a few other parameters among which the quality of the variability " $q$ ". We identified as the main period the value corresponding to the highest value of " $q$ " among the thirty different determinations. We noticed that at the first passage through the periodic detection algorithm the dominant period did not give, in some cases, a sufficiently defined phase-curve (distribution of the measurements folded on a given period). By restricting or enlarging the range of searching periods it was always possible to optimize the phase curve to a certain period. Therefore we decided to execute the algorithm more times by changing the period searching window. The reliability of the assigned period was then determined by looking at the phase curve of each source. Sources of regular variability and with a large amplitude of variability have a clean phase plot with a large $S / N$ ratio that allows the variation of the position of the maximum or minimum brightness and/or the presence of bumps to be distinguished (sometimes identified as the secondary period). In other cases the $S / N$ was not good enough to detect these secondary variations but the phase-curve was clear enough to confirm the main period. We then introduced the following criteria to flag the sources in each band: Flag $=0$ for sources that do not have data points in a given MACHO band; Flag $=5$ for sources with $q \leq 15$ or a period that coincides with the extremes of the searching window in all 30 cases (i.e. sources with a period of 10 or $100,200, \ldots$ 2900,3000 days). Their light curve is judged to be of poor quality. Flag $=1$ and Flag $=2$ are for sources with $q>15$ and for which the assigned period $(P)$ differs by not more than
10 days from the mean of all the measurements (among the thirty possible) with $q>15$ in the blue and red band width, respectively. With these criteria we are confident that a single periodicity is the dominant source of variation in the light-curve. Flag $=6$ is for sources with $q>15$ and a difference from the same mean larger than 10 days. These sources are likely to have more than one significant periodicity. In this work we are not aiming at a detailed analysis of the secondary periods which will be discussed, together with the comparison of LPVs with an ISO counterpart between the Magellanic Clouds and the Galaxy, in Glass et al. (in preparation). Aliases were identified from the diagram $\log (P)$ versus $K_{S}$ as those periods creating clear vertical patterns. These correspond to periods equal to 29 days and in the range from 340 to 390 days, included. These sources were assigned Flag $=9$. Examples of MACHO light-curves for LPVs can be found in Wood et al. (1999). Table 3 summarises the detected periodicities. It lists the description of the Flag (Col. 1), the value of the flag and the number of sources corresponding to a given flag in the red (Cols. 2 and 3) and blue bandwidths (Cols. 4 and 5).

Table 4 provides the magnitudes from the different databases, the bolometric magnitude (see next section) and the parameters that describe the dominant pulsation of all the sources in the sample with an extracted light-curve (458). This table is also available electronically at the CDS and the first 10 lines are given as an example (Table 4). The content of the table is the following: ISO-MCMS name (Col. 1), IJK $K_{S}$ DCMC magnitudes (Cols. 2-4), JHK $K_{S}$ MASS magnitudes (Cols. 57), $L W 2$ and $L W 10$ ISO magnitudes (Cols. 8 and 9), the apparent de-reddened bolometric magnitude (Col. 10), period, amplitude, flag and quality parameter derived for the lightcurve in the MACHO-red bandwidth (Cols. 11-14) and in the MACHO-blue bandwidth (Cols. 15-18).

Values of 99.99 for the magnitudes and of -99 for period and amplitude indicate missing data. Delmotte et al. (2002) have shown that $J_{\mathrm{DCMC}}=J_{2 \mathrm{MASS}}-(0.11 \pm 0.06)$ and $K_{\mathrm{sDCMC}}=$ $K_{\text {s2MASS }}-(0.14 \pm 0.05)$ but we did not apply this correction to Table 4. Amplitudes (A) are the difference between the minimum and the maximum value of the MACHO intensity and are expressed in mag $\times 100$. In a handful of sources this calculation might be biased by spurious data points with a magnitude excursion compared to the sinusoidal behaviour of the lightcurve. Maybe these are real points and represent a flare. The period is in days.

\subsection{Bolometric magnitude}

With the availability of magnitudes from the $I$ band to the mid-IR band $L W 10$, it is possible to determine quite precisely the bolometric magnitude of the sources in the sample by integrating under the spectral energy distribution (SED). This integration has been done by fitting a spline to the SED profile in the plane $\left(v, F_{v}\right)$, extrapolating linearly to zero flux at zero frequency, and also extrapolating linearly at the other extreme of the SED through the two near most points. The SED of all the sources detected by DENIS and ISO were inspected oneby-one to exclude those that are either too red or too blue to describe satisfactorily their SED with the available measurements. 
Table 1. Extract of the table of MACHO counterparts of ISO-MCMS SMC sources.

\begin{tabular}{ccccrcc}
\hline \hline RA & DEC & field & tile & seq. & dist. & ISO - MCMS \\
\hline $0: 48: 44.26$ & $-73: 21: 22.16$ & 212 & 15903 & 16 & 0.22 & J004844.4 - 732119 \\
$0: 48: 44.67$ & $-73: 17: 55.01$ & 212 & 15904 & 27 & 1.02 & J004844.5 - 731754 \\
$0: 48: 44.29$ & $-73: 20: 19.91$ & 212 & 15904 & 5 & 2.36 & J004844.7 - 732018 \\
$0: 48: 49.26$ & $-73: 16: 25.11$ & 212 & 15905 & 525 & 1.12 & J004849.4 - 731627 \\
$0: 48: 49.91$ & $-73: 20: 04.07$ & 212 & 15904 & 6 & 1.08 & J004849.8 - 732002 \\
$0: 48: 51.10$ & $-73: 21: 40.58$ & 212 & 15903 & 11 & 0.71 & J004850.7 - 732138 \\
$0: 48: 51.75$ & $-73: 22: 39.51$ & 212 & 15903 & 1 & 0.55 & J004851.8 - 732239 \\
$0: 48: 54.13$ & $-73: 15: 58.40$ & 212 & 15905 & 523 & 1.43 & J004854.0 - 731557 \\
$0: 48: 59.26$ & $-73: 11: 53.24$ & 212 & 15906 & 2 & 1.26 & J004859.7 - 731155 \\
$0: 49: 0.221$ & $-73: 22: 23.62$ & 212 & 15903 & 9 & 0.71 & J004900.4 - 732224 \\
\hline
\end{tabular}

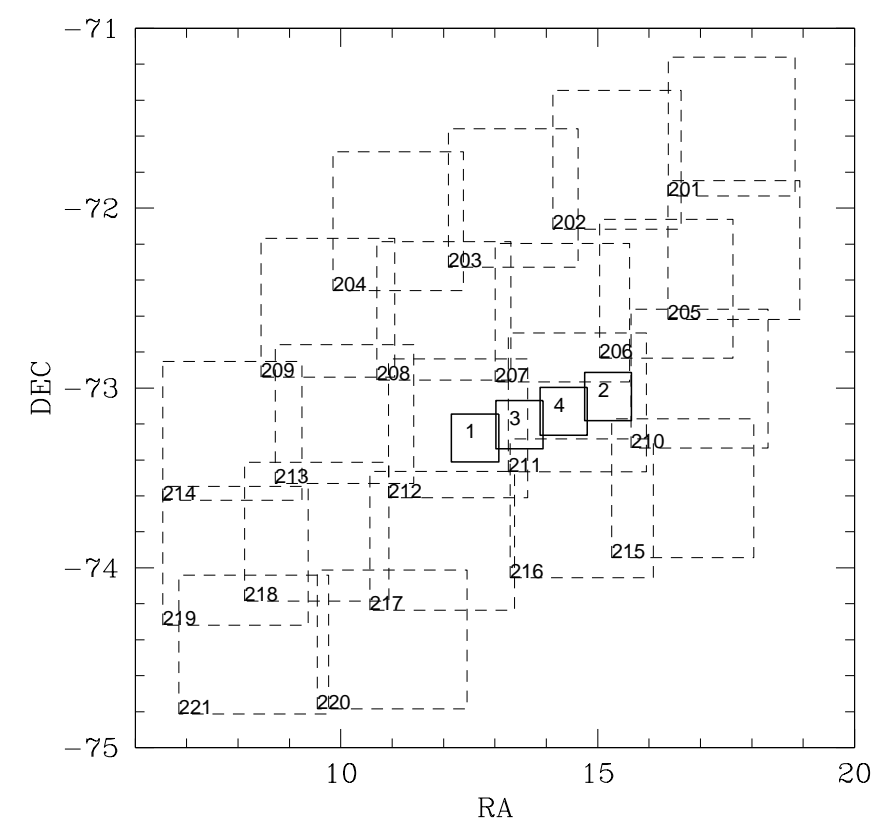

Fig. 1. Location of the fields observed by ISO (continuous line) and MACHO (dashed line) in the SMC. The ISO fields lie in the bar of the galaxy and are each $16^{\prime} \times 16^{\prime}$ in size. Both right ascension (RA) and declination (DEC) are in degrees.

The adopted extrapolations represent the SED very well, almost independently of the circumstellar opacity for all $\mathrm{M}$ spectral subtypes (Loup et al. in preparation). That article gives a detailed determination of the multi-band bolometric correction for AGB stars by combining data from the major survey instruments: DENIS, 2MASS, ESO, IRAS, ISOCAM and MSX. The bolometric correction is obtained from the different combination of pass-bands and a theoretical model for the SED. This is compared to the correction obtained from the integration under the SED using a spline or a linear fit through the flux points. A fairly well sampled SED that detects most of the energy emitted by the objects in a set of characteristic bands is a necessary condition to derive a good bolometric luminosity $\left(M_{\mathrm{BOL}}\right)$ for stars of different $\mathrm{M}$ spectral subclass and $\mathrm{C}$ stars.

The best wavelength coverage for the data in the sample studied here is obtained by combining DENIS and ISO magnitudes. Though 2 MASS $K_{S}$ magnitudes exist for 455 sources,

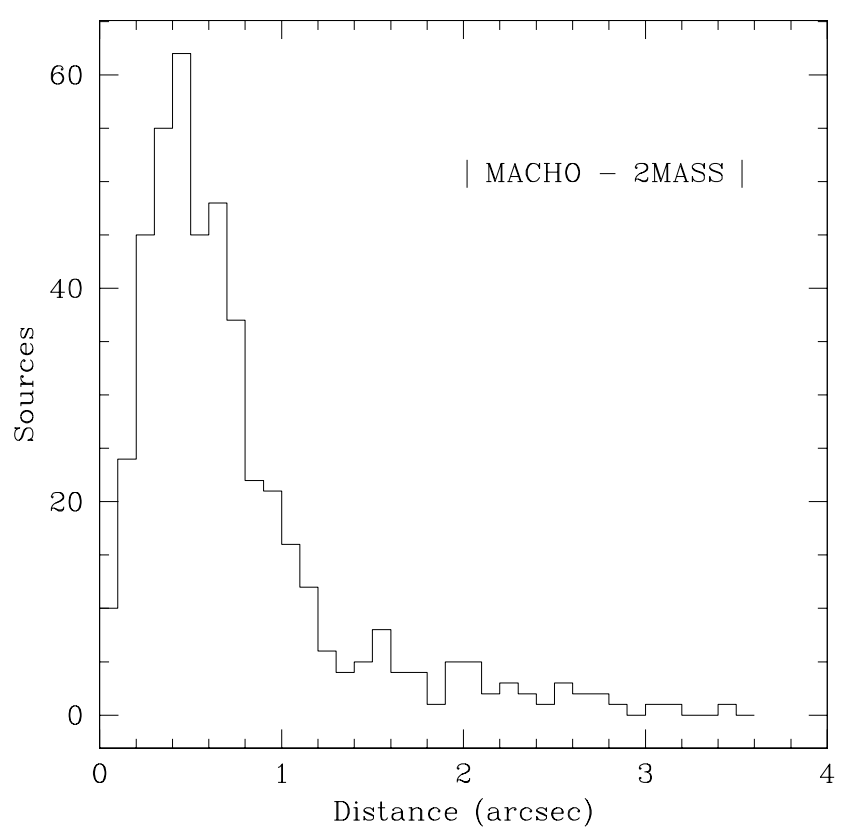

Fig. 2. Histogram of the absolute distance between the 2MASS counterpart of an ISO-MCMS source and the corresponding MACHO source.

DCMC- $J K_{S}$ magnitudes exist for 366 sources and DCMCIJ mag exist for about 450 sources. On the one hand it would seem natural to combine $I J$ DENIS magnitudes with 2MASS$K_{S}$ magnitudes and the ISO magnitudes, for the same sources, to derive the bolometric magnitude. On the other hand the DCMC-IJK $K_{S}$ magnitudes were obtained by simultaneous observations and this is a unique constraint for the SED profile.

Note that the amplitude of variation in the mid-IR bands can be as high as 1 mag (e.g. van Loon et al. 1998; van Loon et al. 2001), this effect may influence the shape of the SED during the variability cycle. The constraint of the $I$ band to the shape of the SED at higher frequencies is very important for optically thin AGB stars because the peak of the SED is at about $K_{S}$. In Loup et al. (in preparation); we show that the linear extrapolation to zero flux at the longer frequencies starting from the flux in the $J$ band subtends a larger area than the extrapolation starting from the flux in the $I$ band. This effect overestimates the bolometric magnitude. 
Table 2. Table of ISO-MCMS sources with a confident DENIS/ 2MASS counterpart but without an associated MACHO light-curve.

\begin{tabular}{|c|c|}
\hline ISO - MCMS & MACHO counterpart? \\
\hline J004850.9- 731402 & $d>17^{\prime \prime}$ \\
\hline J004902.7 - 732141 & $d>5^{\prime \prime}$, only 3 data points \\
\hline J004907.2 - 731335 & $d>12^{\prime \prime}$, too few data points \\
\hline J004907.8 - 730920 & $d>14^{\prime \prime}$, too few data points \\
\hline J004908.2 - 731415 & $d>14^{\prime \prime}$ \\
\hline J004909.5 - 731133 & $d>7^{\prime \prime}$ \\
\hline J004910.5 - 731859 & $d>3^{\prime \prime}$, too few data points \\
\hline J004934.4 - 731409 & $d>9^{\prime \prime}$ \\
\hline J004940.8 - 731352 & $d>15^{\prime \prime}$, too few data points \\
\hline J004940.9 - 731412 & $d>8^{\prime \prime}$ \\
\hline J004959.0 - 731058 & $d>7^{\prime \prime}$ \\
\hline J005001.7 - 731349 & $d>9^{\prime \prime}$ \\
\hline $\mathrm{J} 005034.2-731354$ & $d>15^{\prime \prime}$ \\
\hline J005108.1 - 731343 & $d>5^{\prime \prime}$ \\
\hline J005117.5 - 731659 & $d>4^{\prime \prime}$, too few data points \\
\hline J005121.1 - 731416 & $d>4^{\prime \prime}$ \\
\hline J005129.7 - 731044 & $d>8^{\prime \prime}$ \\
\hline J005131.4 - 732008 & $d>5^{\prime \prime}$, too few data points \\
\hline J005140.2 - 731333 & \\
\hline J005202.5 - 731339 & $d>6^{\prime \prime}$ \\
\hline J005207.0 - 731407 & $d>7^{\prime \prime}$ \\
\hline J005211.5 - 731351 & $d>16^{\prime \prime}$ \\
\hline J005217.8 - 731327 & $d>4^{\prime \prime}$ \\
\hline J005218.9 - 730921 & $d>4^{\prime \prime}$, too few data points \\
\hline J005230.8 - 730259 & $d>8^{\prime \prime}$, too few data points \\
\hline J005239.9 - 730912 & \\
\hline J005245.7 - 730425 & $d>6^{\prime \prime}$ \\
\hline J005302.9 - 730746 & $d>6^{\prime \prime}$ \\
\hline J005306.4 - 730633 & $d>10^{\prime}$ \\
\hline J005309.0 - 730404 & $d>9^{\prime \prime}$ \\
\hline J005309.9 - 731324 & $d>3^{\prime \prime}$, too $\mathrm{f}$ \\
\hline J005310.3 - 730722 & $d>3^{\prime \prime}$, too few data points \\
\hline J005405.7 - 730459 & $d>3^{\prime \prime}$, too few data points \\
\hline J005417.7 - 730504 & $d>3^{\prime \prime}$, poor sampling \\
\hline J005418.1 - 730523 & $d>7^{\prime \prime}$ \\
\hline J005518.6 - 731420 & $d>2^{\prime \prime}$, too few data points \\
\hline J005549.0 - 730257 & $d>4^{\prime \prime}$, too few data points \\
\hline J005646.4 - 730519 & $d>12^{\prime \prime}$ \\
\hline J005648.0 - 730404 & $d>5^{\prime \prime}$ \\
\hline J005650.1 - 731040 & $d>2^{\prime \prime}$, too few data points \\
\hline J005650.9 - 730420 & $d>7^{\prime \prime}$ \\
\hline J005749.0 - 730522 & $d>11^{\prime \prime}$, too few data points \\
\hline J005822.0 - 730734 & $d>4^{\prime \prime}$ \\
\hline J005833.1 - 730528 & $d>16^{\prime \prime}$ \\
\hline
\end{tabular}

Prior to the calculation of the flux emission in the different bands and the integration under the SED we de-reddened the data adopting the extiction law by Glass (1999). For the DENIS pass-bands we used $\left[A_{V}: A_{I}: A_{J}: A_{K_{S}}=1: 0.592: 0.256\right.$ : 0.089 ], and we assumed zero absorption in the ISO wave bands. We used $R_{V}=3.1$ and $E(B-V)=0.065$, the latter is the average of the measurements, in the SMC, discussed in Westerlund (1997). The zero-point of the bolometric scale is computed with: $M_{\mathrm{BOL}, \odot}=4.74$ and $f_{\mathrm{tot}, \odot}=1.371 \times 10^{-6} \mathrm{erg} \mathrm{cm}^{-2} \mathrm{~s}^{-1}$. A comparison of our bolometric magnitudes with the relations obtained by Alvarez et al. (2000) and Montegriffo et al. (1998) is given in Appendix B.

\section{Discussion}

\subsection{Period-luminosity relation(s)}

Figure 3a shows the relation between the logarithm of the period (days) and $K_{S}$. Variable stars are distributed on four different parallel relations. These relations were first discovered by Wood et al. (1999) from the analysis of a sample of MACHO stars in the LMC. They were subsequently found by Cioni et al. (2001) using EROS2 (Lasserre et al. 2000) light-curves, by Noda et al. (2002) using the MOA database and by Lebzelter et al. (2002) using the AGAPEROS data. These latter measurements refer also to samples of stars in the LMC. A preliminary comparison between the relations in the LMC and in the SMC has been shown by Cioni et al. (2002a), concluding that sources in both galaxies occupy the same relations in the diagram $\left(K_{S}\right.$, $\log (P))$.

There are a few differences that depend on the way the two samples were selected and the light-curves extracted: in the SMC there are sources with longer periods because the MACHO data cover a longer time range than the EROS2 data. Thus it is possible to identify longer periodicities. Also there is a relative lack of SMC sources around the tip of the red giant branch (TRGB) because the LMC sources were selected from the DCMC and not among those sources detected at 7 and $12 \mu \mathrm{m}$. It may be that AGB stars that are not yet in the TPAGB phase or are in a very early phase of their TP-AGB evolution have a mid-IR emission below the ISO detection limit. The ISO survey was indeed designed to find mass-loss, not to detect sources without circumstellar extiction.

The analysis of the light-curves of LMC sources detected by ISO has just started. The $K_{S}$ magnitudes are always a oneepoch measurement, and coupled with the amplitude of variation, that in the $K_{S}$ band can be as high as $1.5 \mathrm{mag}$ for $\mathrm{OH} / \mathrm{IR}$ stars, prevent us from detecting a difference (if any) in the width of the PLRs due to metallicity. The same situation holds for stars in the Bulge (Alard et al. 2001), which have the additional disadvantage that the distance to the AGB stars is too uncertain to find all four PLRs. Furthermore, AGB stars in stellar clusters are too few to outline the presence of one and possibly two PLRs. There is evidence that in the elliptical galaxy NGC 5128 one and maybe two PLRs exits for LPVs (Rejkuba 2002).

The atmosphere of AGB stars is dominated by molecules of $\mathrm{TiO}$ and $\mathrm{VO}$ if the star is $\mathrm{O}-$ rich $(\mathrm{C} / \mathrm{O}<1)$ or by molecules of $\mathrm{CN}, \mathrm{C}_{2}$ and other carbonaceous molecules if the star is $\mathrm{C}$-rich $(\mathrm{C} / \mathrm{O}>1)$. Stars of both types are easily statistically distinguished in the $\left(J-K_{S}, K_{S}\right)$ colour-magnitude diagram (Fig. 4a) because of the different strength of the molecular absorption bands at these particular wavelengths (Cioni et al. 2001; Loup et al. 2002). The dividing line at $\left(J-K_{s}\right)=1.33$ takes into account the shifts calculated by Delmotte et al. (2002) between 2MASS and DCMC magnitudes and has been defined in Cioni \& Habing (2003) for the whole AGB population of the SMC. It was also formerly checked by Cioni et al. (2000) via the 
Table 3. Summary of detected periodicities.

\begin{tabular}{|c|c|c|c|c|}
\hline Description & & ${ }_{\mathrm{D}}^{N}$ & & ${ }_{\text {UE }}^{N}$ \\
\hline No curve & 0 & 8 & 0 & 9 \\
\hline Good & 1 & 96 & 2 & 91 \\
\hline Poor & 5 & 103 & 5 & 208 \\
\hline Multi - Periodic & 6 & 91 & 6 & 91 \\
\hline Alias & 9 & 160 & 9 & 59 \\
\hline
\end{tabular}

cross-identification with the large sample of spectroscopically identified C-rich stars of Rebeirot et al. (1983). Filled symbols in Fig. 3 indicate $\mathrm{C}-$ rich objects. In Fig. $3 \mathrm{~b}$ they define relation $\mathrm{B}$ and $\mathrm{C}$, and dominate relation $\mathrm{D}$ (possibly offset from the oxygen-rich stars).

\subsection{Mass and age}

According to the Vassiliadis \& Wood (1993) prescription for the evolution of low- and intermediate-mass stars, carbon stars are less massive than about $3 M_{\odot}$. They form after a certain number of shell flashes when the atoms of carbon outnumber the oxygen atoms. Hot bottom burning may prevent massive stars from becoming C-rich. Each track drawn in Fig. 3b starts at the first shell-flash cycle on the TP-AGB phase. Most of the $\mathrm{C}$-rich stars in the SMC sample have a mass between 1 and $3 M_{\odot}$. The brightest stars $\left(M_{\mathrm{BOL}}<-7\right)$ are probably supergiants (Wood et al. 1992). The most massive AGB stars with a mass of about $5 M_{\odot}$ are about $0.1 \mathrm{Gyr}$ old. The oldest detected AGB stars are about 9 Gyr old, but most of the sample is from 0.6 to 2 Gyr old.

In the LMC (Cioni et al. 2001) C-rich stars occupy only the brightest part of the PLRs, thus in a lower metallicity environment, such as that of the SMC, C-rich stars form at lower masses, unless these stars are in the luminosity dip that follows a thermal pulse or at the minimum of their light-curve. Menzies et al. (2002) recently showed that the top magnitude of the AGB stars in $K_{S}$ in the dwarf galaxy Leo I $([\mathrm{Fe} / \mathrm{H}]=-2.0)$ are mostly carbon stars. They have $J-K_{S}>1.1$. The value of the $J-K_{S}$ that discriminates between O-rich and C-rich stars is a function of metallicity. The lower the metallicity, the bluer the colour. Obscured AGB star candidates $\left(J-K_{S}>2\right)$ might also be of $\mathrm{C}$ type (50\% level) and the observation at two different epochs indicates that at least one of these object might be a Mira variable $\left(\Delta K_{S}=0.87\right)$.

\subsection{Large amplitude variables}

Figure $3 \mathrm{c}$ shows the amplitude of the light-curves as a function of $M_{\mathrm{BOL}}$ for $\mathrm{C}$-rich and $\mathrm{O}-$ rich objects. The sources with $A * 100>110$ and $f=1$ have $q>100$ and are distributed in the PLR(s) as shown in Fig. 3d. They occupy relation C which is the location of Mira variables in the LMC and the Galaxy. Overplotted is the sequence derived by Feast et al. (1989) and the single LPVs (crosses) as re-analysed in this work (Appendix A). This sets the minimum level of the quality parameter for Mira variables. This sequence was obtained including both $\mathrm{O}$-rich and C-rich AGB stars with a period $P<420$ days. A distance modulus of 18.99 has been used (Cioni et al. 2000). The single star at $\log (P)=2.1$ and $M_{\mathrm{BOL}}=-6$ is of large amplitude because of flare-like events superimposed on a regular variation of smaller amplitude, thus this source should not be regarded as a Mira star.

The scatter in the PLR of the sources in this study results from the unavailability of bolometric light-curves. However, it is consistent with the sigma associated with the relation derived by Feast et al. (1989). Estimating the magnitude of this effect would be valuable for studies that plan to use AGB stars as tracers of the star formation history of systems as far as $250 \mathrm{kpc}$ (Kucinskas et al. 2002). This preliminary investigation on the use of AGB stars concerns mainly early-type AGB stars which vary with amplitudes much below that of Mira-type stars. On the other hand, Kucinskas et al. combined broad-band observations from the literature. It should not be neglected that the amplitude of variability of AGB stars is different in each photometric band. Only simultaneous observations in different bands, such as those that will be available with GAIA, provide a coherent SED from which a single-epoch bolometric magnitude can be derived.

Finally we conclude that a large amplitude and a large value of $q$ indicate a clear and regular light-curve; thus a Mira star. There are some sources of large amplitude and with a large value of the quality parameter suspected of multi-periodicity. Their main period does not correspond to relation C. A few Mira variables in sequence D have been found by Alard et al. (2001). On the contrary, this PLR could be populated by binary stars with an AGB companion (Wood et al. 1999). In what follows we mainly discuss the properties of the stars with a light-curve in the red band of MACHO and $f=1$ or $f=6$.

\subsection{Mira and Semi-Regular variables}

The distinction between Mira and Semi-Regular (SR) stars has become more and more subtle with the availability of more sensitive long term observations and large samples of stars. The stellar light-curve can be as regular in SR as in Mira stars. Lebzelter et al. (2002) define a classification criterium for LPVs based only on the regularity and multi-periodicity of the light-curves. Most of the Mira stars fall into the class of regular variables but not all regular variables have large amplitudes of variation. SR belong to a second class and have on average a smaller amplitude than the stars in the first class. Into the third class fall irregular variables that do not show a clear periodicity. The Mira phase is believed to be just a moment during the AGB evolution and the star can thus become or have been SR. This scenario is supported by observations of very nearby variable stars over a period of about 90 years (i.e. Percy \& Bagby 1999; Kiss et al. 2000). However the evidence, e.g. from globular clusters, suggests that most SRs are in the pre-Mira stage.

Mira variables lie on one PLR (Feast et al. 1989) and SR occupy this and other PLRs according to their mode of pulsation (Wood et al. 1999; Cioni et al. 2001; Noda et al. 2002; 
Table 4. Extract of the table of the properties of LPVs with ISO counterpart in the SMC.

\begin{tabular}{|c|c|c|c|c|c|c|c|c|c|c|c|c|c|c|c|c|c|}
\hline ISO - & I & $\begin{array}{c}J \\
\text { DCMC }\end{array}$ & $K s$ & $J_{2}$ & $\begin{array}{c}H \\
\text { 2MASS }\end{array}$ & $K s$ & IS & & $m_{\mathrm{bol}_{0}}$ & & & & $q$ & & $\begin{array}{c}A \\
-\mathrm{BL}\end{array}$ & & $q$ \\
\hline J004844.4-732119 & 14.69 & 12.91 & 99.99 & 13.09 & 12.11 & 11.80 & 10.96 & 9.84 & 99.99 & 203 & 428 & & 70 & 374 & 74 & 9 & 67 \\
\hline & .65 & 82 & & & & & & & & 43 & 91 & & & 342 & 180 & & 277 \\
\hline & 9.65 & 57 & .99 & & & & 76 & 06 & & 2900 & 401 & & 20 & 900 & 334 & 5 & 15 \\
\hline 1627 & 14.29 & 13.99 & 99.99 & 14.19 & 14.09 & 13.77 & 11.64 & 10.91 & & 1900 & 95 & & 6 & 2063 & 82 & 6 & 116 \\
\hline 2002 & 11.80 & 10.31 & 99.99 & 10.48 & 9.65 & 9.42 & 9.26 & 9.24 & 99. & 29 & 220 & & 10 & 821 & 226 & 5 & 10 \\
\hline & 14.68 & 13.67 & 12.68 & 3.80 & 13.15 & 13.05 & 11.77 & 9.99 & & 368 & 24 & & 31 & 1667 & 42 & 5 & 11 \\
\hline & 10.90 & 9.45 & & & 8.83 & 8.57 & 8.04 & 7.52 & & 352 & 456 & & 6 & 350 & 301 & 9 & 7 \\
\hline & 3.62 & 12.37 & 99.99 & 12.59 & 11.85 & 11.69 & 11.58 & 99.99 & & 10 & 19 & & 88 & 10 & 21 & 5 & 19 \\
\hline 1155 & 13.04 & 11.80 & 99.99 & 11.96 & 11.17 & 11.00 & 10.74 & 99.99 & 99. & 368 & 184 & & 1 & 21 & 29 & 5 & 4 \\
\hline J004900.4 - 732224 & 14.16 & 12.47 & 99.99 & 12.65 & 11.70 & 11.35 & 10.74 & 99.99 & 99.99 & 239 & 67 & & 137 & 237 & 99 & 22 & 207 \\
\hline
\end{tabular}
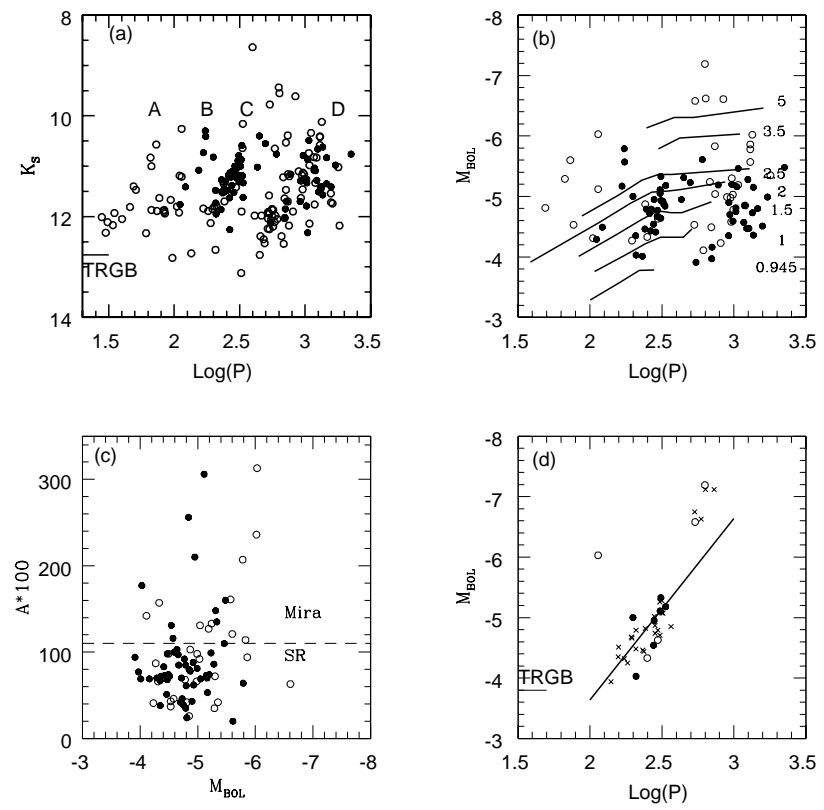

Fig. 3. Diagrams for the sources in the sample with a MACHORED light-curve and $f=1$ or $f=6$. a) $K_{S}$ versus $\log (P)$ relation. Letters $\mathrm{ABCD}$ identify the parallel sequences as defined in Wood et al. (1999). Filled symbols indicate probable C-rich objects. b) PLRs and overplotted theoretical tracks by Vassiliadis \& Wood (1993). The numbers indicate the initial mass of the stars in each track. c) Amplitude distribution as a function of bolometric magnitude. d) PLRs for sources with an amplitude above the dashed line indicated in c), $q>100$ and $f=1$. The continuous line is the Mira PLR fitted through the crosses corresponding to $P<420$ days obtained by Feast et al. (1989); a distance modulus of $m-M=18.99$ (Cioni et al. 2000) has been applied. Symbols identify O-rich stars (open circles), C-rich stars (filled circles) and Feast et al. (1989) variables (crosses).

Lebzelter et al. 2002). If Mira variables are selected as discussed in Sect. 4.3 then they are clearly concentrated on the C PLR. This selection criterium is based on the amplitude of variation. However, the sources are the most regular as well $(q>100)$.

Whitelock et al. (1997), by analysing the light-curves of a dozen C-rich AGB stars in the Milky Way Galaxy, showed that the distinction between Mira and SR variables is not necessarily very clear among carbon stars, and may not be useful as a distinction for C-rich stars as it is for O-rich stars. Figure $4 \mathrm{~b}$ shows the histogram of the distribution of the amplitude of variation separately for O-rich and C-rich stars. Perhaps there is a distinction between Mira, the pulsators at the tail of the amplitude distribution(s), and SR. Both O-rich and $\mathrm{C}$-rich sources have a histogram with a dominant peak at about $A \times 100=40$ and $A \times 100=80$, respectively, and a tail to larger amplitudes. The fact that $\mathrm{C}$-rich stars have on average larger amplitudes than $\mathrm{O}$-rich stars is different from what was found in the LMC. There, both histograms had the same distribution (Fig. 4 in Cioni et al. 2001). The same histogram but for the amplitudes derived from the BLUE light-curve shows that $\mathrm{O}-$ rich stars peak at about $A * 100=50$ and C-rich stars at about $A * 100=100$. This confirms that the average amplitude is larger in the blue than in the red spectral domain.

\subsection{Periodicity peaks}

The histogram of the distribution of the variable sources versus their period is shown in Fig. 4c. The distribution of O-rich stars has a main peak at roughly $\log (P)=2.80$ and two lower peaks at about $\log (P)=1.95$ and $\log (P)=2.30$. C-rich stars have well defined peaks at about $\log (P)=2.45$ and $\log (P)=3$. Note that most of the sources with $2.53<\log (P)<2.59$ have been excluded because their period determination is suspected of aliasing.

Figure 4 in Cioni et al. (2001) shows that O-rich AGB stars in the LMC have a strong broad peak between $\log (P)=1.5$ and 2.0, and a lower peak at about $\log (P)=2.45$, while C-rich AGB stars are homogeneously distributed from $\log (P)=1.5$ to 2.5. The first LMC peak of O-rich AGBs is probably a consequence of the selection criterium used to define the two samples. The SMC sample is extracted from sources with an emission in the mid-IR, these stars are brighter and with longer periods than the stars in the LMC sample which were selected from the DCMC database. The difference in strength is probably due to the mean difference in the AGB type between the two Clouds. There are more carbon stars in the SMC which is on average more metal poor than the LMC (Cioni \& Habing 2003). This effect is also important for the sources populating relation $\mathrm{C}$ which are predominantly $\mathrm{C}$-rich in the SMC and associated to the peak at about $\log (P)=2.45$. That the majority 
of accepted Miras in the SMC are carbon stars was first recognized by Lloyd Evans et al. (1988) as the result of a search for large-amplitude red variables in the Radcliffe Variable Star Field. The longer time range spanned by the MACHO observations allows to detect periodicities above $\log (P)=2.8$ which was about the limit reached in the LMC study using EROS light-curves. The large number of sources detected in the SMC at these periods are likely to be multi-periodic. Their secondary period will be part of relation $\mathrm{A}, \mathrm{B}$ or $\mathrm{C}$, thus they will enhance the number of O-rich stars between $\log (P)=1.5$ and 2.5. In more detail we found that in our sample, among those with a red light-curve and $q>15,24$ sources have flag $=6$. These sources have $2.0<\log (P)<2.3$ which, taking into account the differences between the various period determinations and the mean, gives a period ratio from 1.5 to 2 .

The same sample of LMC stars analysed by Cioni et al. (2001) was afterwards studied by Lebzelter et al. (2002) using the AGAPEROS datasets. Ninety per cent of their sources were classified into: regular, SR, irregular and other variability classes. Their analysis covers a time window of up to 900 days and is thus incomplete for the longest periods, but it is more complete for sources with an amplitude of variation below $0.1 \mathrm{mag}$. The authors found that the period distribution of regular variables has two peaks: one at $\log (P)=2.0$ and the other at $\log (P)=2.5$. The latter is probably formed by Mira stars. SR variables peak at about $\log (P)=1.8$. The peak at the shortest periods is probably the same as the one found here. The peak of mostly Mira stars corresponds to the location of the peak of the C-rich SMC stars. In the Baade's window, Glass \& Schultheis (2002) found that most of the latetype $\mathrm{M}$ giants have $\log (P)<2.0$; those few with a secondary longer period have about $\log (P)>2.4$. The histogram that can be derived from their Fig. 2 is very similar to that of $\mathrm{O}$-rich stars in the SMC (Fig. 4c).

\section{6. $\left(J-K_{S}, K_{S}\right)$ diagram}

In a similar fashion to the LMC sample (Fig. 7 in Cioni et al. 2001), variable stars of a different type and variability class are plotted in the colour-magnitude diagrams (CMDs) in Fig. 5. The distinction has been made as follows: Miras have $A \times 100>110$ independently of their type, and a regular lightcurve with a well defined single periodicity. Similar sources but with a smaller amplitude of variation are SRa stars. SRbs are those stars which indicate the presence of a secondary period. Note that in this last category there is no distinction based on the amplitude of variation. As in the LMC, Mira stars are uniformly distributed in the regions where optically thin $\mathrm{O}_{-}$ rich and C-rich, and also more optically thick stars, are located (Fig. 5a). SRa and SRb are almost equally distributed within the two branches of $\mathrm{O}$-rich and $\mathrm{C}$-rich optically thin AGB stars. Figure 5d shows the distribution of the sources that could not be classified in one of the three categories either because the light-curve has a too low $S / N$ or it is extremely irregular. Most of these stars (180 out of 194) have $A>10$, thus they are variable stars and lie above the TRGB, $K_{S}=12.62$ (Cioni et al. 2000). It is possible that stars considerably brighter
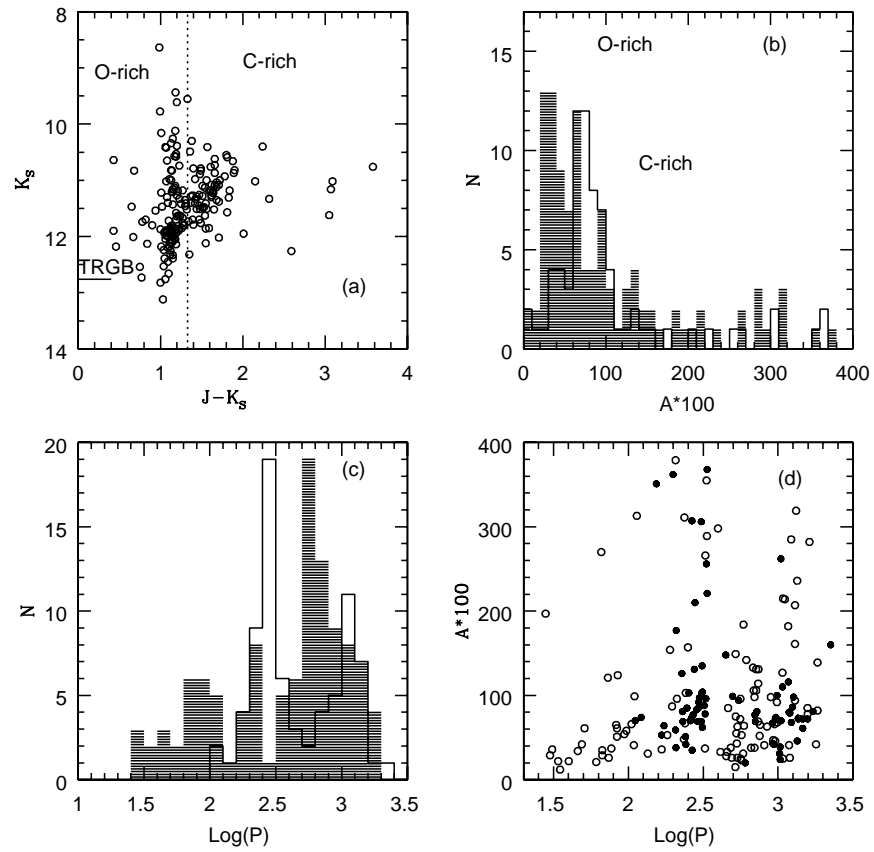

Fig. 4. Variable $\mathrm{O}-$ and $\mathrm{C}-$ rich AGB stars a). The histogram of the amplitude of variation $\mathbf{b}$ ) and that of the period distribution c) are shown for sources of both types. The amplitude as a function of period is shown in d).

than $K_{S}=10$ are supergiants and that fainter stars at about $\left(J-K_{S}\right)=0.4$ are foreground objects. Schultheis et al. (2000) found variable star candidates at $\left(J-K_{S}\right)<1$. They conclude that it is probably a result of uncertainties in the determination of extinction. However, they find a displacement of the Bulge stars detected as variables in both $J$ and $K_{S}$ to bluer colours than the sequence indicated by all detected ISO stars in the diagram $(L W 2-L W 3, L W 3)$. It would be interesting to know if some of these stars are supergiants and of which type.

Finally we conclude that all the sources in the sample are variable stars because they have amplitudes above or equal to 0.1 mag either in the RED or BLUE wave band. A similar conclusion was reached by Alard et al. (2001) for a sample of sources in Baade's window detected at 7 and $15 \mu \mathrm{m}$. The emission at the longest wavelength is a signature of mass-loss rate. Using their dependence between $M_{\mathrm{BOL}}$ and the mass-loss rate and assuming that the mass-loss rate of highly evolved AGB stars does not depend very strongly on metallicity (van Loon 2000), we derive that the sources in the present sample lose mass at a rate from $10^{-7} M_{\odot} \mathrm{yr}^{-1}\left(M_{\mathrm{BOL}}=-4\right)$ to $10^{-4} M_{\odot} \mathrm{yr}^{-1}\left(M_{\mathrm{BOL}}=-7\right)$, but most of the objects have $M_{\mathrm{BOL}}=-5$ which corresponds to a mass-loss rate of about $10^{-6} M_{\odot} \mathrm{yr}^{-1}$. Note that a complete study of the dependence of mass-loss includes temperature, mass and outflow velocity as well.

\section{7. $\left(K_{S}-L W 10, L W 10\right)$ diagram}

The $7 \mu \mathrm{m} L W 2$ filter does not include a large dust contribution for non-Mira sources and it is mostly a measure of the photospheric emission. On the other hand, for stars with a 
relatively thick circumstellar dust envelope, this band is strongly affected by the presence of dust (Alard et al. 2001; Omont et al. 1999). The $12 \mu \mathrm{m} L W 10$ filter, which is very similar to the IRAS $12 \mu \mathrm{m}$ band, includes the $9.7 \mu \mathrm{m}$ silicate and the $11.3 \mu \mathrm{m} \mathrm{SiC}$ dust features. Thus the colour $(K-L W 10)$ provides a strong indication of the amount of circumstellar dust for the entire sample.

Figure 6 shows the colour magnitude diagram $\left(K_{S}-L W 10\right.$, $L W 10$ ) for the sources in the present sample, distinguishing between Mira and SR variables. Except for a few SR all the sources with $\left(K_{S}-L W 10\right)>2$ are Mira variables (Fig. 6a). From the location of some $K-$ and M-type supergiant (SG) stars in the ISO-MCMS one derives that they have $\left(K_{S}-L W 10\right)<1$ and $L W 10=8$. They follow a sequence that extends almost vertically to fainter magnitudes, but the nature of those sources with $L W 10>10$ is not confirmed. Bright O-rich LPVs would have $\left(K_{S}-L W 10\right)=3$ and $L W 10=7$. These stars would have a late $M$ subtype and not many of them are expected in the SMC. A quantitative statement will be possible once the study of the LMC LPVs detected by ISO is completed. A few SRb stars populate the fainter part (Figs. 6b,c). There are many supergiant stars along the whole sequence that were not classified in any of the three categories (Fig. 6d). It is thus not surprising that their light-curve is more difficult to classify because of a low $S / N$ and irregularities. Thus, except for a few stars overlapping the region occupied by SR and Mira stars we are able to distinguish sources with a poor or nonregular light curve. A more careful look at the near-IR colourmagnitude diagram (Fig. 5) confirms that the unclassified stars with $\left(J-K_{S}\right)=1$ or a bluer colour are experiencing the supergiant phase because AGB stars have overall $\left(J-K_{S}\right)>1$. This is quite a good criterion to discriminate between supergiant and AGB stars, though the distinction is better achieved in the combined near- and mid- infrared diagram (Fig. 6).

Most carbon stars are located at about $\left(K_{S}-L W 10\right)=1$ and $8<L W 10<11$ (Fig. 6 - filled points); the sequence of dust obscured C-type stars extends to redder colours but the $L W 10$ mag follows a shallower slope such as $\left(K_{S}-L W 10\right)=4$ at $L W 10=8$. On average $\mathrm{C}$-rich stars are redder than $\mathrm{O}$-rich stars.

Planetary nebulae and emission line objects, included in the ISO-MCMS catalogue, also have red colours $\left(K_{S}-L W 10>\right.$ 2) but they have fainter magnitudes with respect to Mira stars and are roughly located in a parallel sequence. Three of these objects are present in Fig. $6 \mathrm{~d}$. The object at $L W 10<8.5$ and $K_{S}-L W 10>4$ is a known carbon star while the object with the same $L W 10$ magnitude and a bluer colour is a known variable $\mathrm{M}$ giant, because of its red colour we erroneously classified it as a carbon star.

\section{8. $\left(K_{S}-L W 2, L W 2-L W 10\right)$ diagram}

Figure 7 shows the colour-colour diagram of the variables identified in this study. The O-rich sources with (LW2 $L W 10)>0.4$ are AGB stars or red supergiants. These AGB stars have a significant circumstellar extinction. This is also the colour of obscured O-rich and C-rich stars, and those with the highest amplitude of variability have $\left(K_{S}-L W 2\right)>1.5$. O-rich stars without a considerable mass loss have approximately $(L W 2-L W 10)=0$ but can have $0<\left(K_{S}-L W 2\right)<1$. There is not a remarkable distinction between SRa and SRb pulsators. At about $\left(K_{S}-L W 2\right)>3.5$ one can find $\mathrm{PN}$ and emission line objects.

It seems (Fig. 7d) that we may have missed the classification of a few Mira variables, O-rich and C-rich SR stars. At this stage it is worth going back to the specific light-curves to find out the reason of this missed classification and to perhaps identify some peculiar objects. Among the objects with $K_{S}-L W 2>1.5$ (Fig. 7d) J005113.6-731035, a known carbon star (RAW 658), has a flat light-curve, while the light-curve of J005136.5-732016, identified as a PN, is irregular. Another PN is J005157.9-731421 which may have a regular light-curve with an amplitude of about $0.8 \mathrm{mag}$. The periodogram of the other objects, J004849.4-731627 (emission line star), J005212.9-730852 (variable M giant) J005304.7-730409 and J010020.6-730648 (BMB-W 29 carbon star) shows a very low amplitude with flare-like events.

\subsection{Miscellaneous}

$\mathrm{SRb}$, as have been defined in this work, do not constitute a separate class of variables. They are clearly multi-periodic objects and from their photospheric and dust colours they do occupy the same regions of SRa and Mira stars. Until a confirmation of the specific theoretical models (such as those developed by Winters et al. 1994 to explain the multi-periodicity of the light-curve variation by coupling of stellar pulsation with the dust formation) or follow up radial velocity observations (to infer the binary nature of the sources) are available, the most appropriate class to which assign these stars is that of general SR variables. This is also strengthened by the fact that most of the objects in the sample indicate the presence of more than one periodicity. A sophisticated observational and theoretical analysis of the multiple periods is required to further address this point. Because we did not discriminate on the amplitude of SRb stars some of them do have Mira-like amplitude but are likely to have more than one significant period.

Most of the DENIS sources in the present sample were observed in December 1996 while 2MASS observations date August 1998. This difference in time of about two years may allow us to recognize variable stars by comparing their detection in the near-IR bands of DENIS and 2MASS. Figure 8 shows the distribution of these differences for all the sources in the sample. We checked that the separate histogram of sources with an assigned MACHO periodicity is the same as that of sources with Flag $=5$ (a period was not assigned but the source is probably a variable). The histogram does not account for the systematic difference between the 2MASS and DCMC magnitudes as measured by Delmotte et al. (2002). Selecting only the variable stars with the largest amplitude produces a similar histogram. The displacement from zero indicates that the sources are variable stars. Note that the difference is approximately the same in the $J$ and $K_{S}$ bands. 

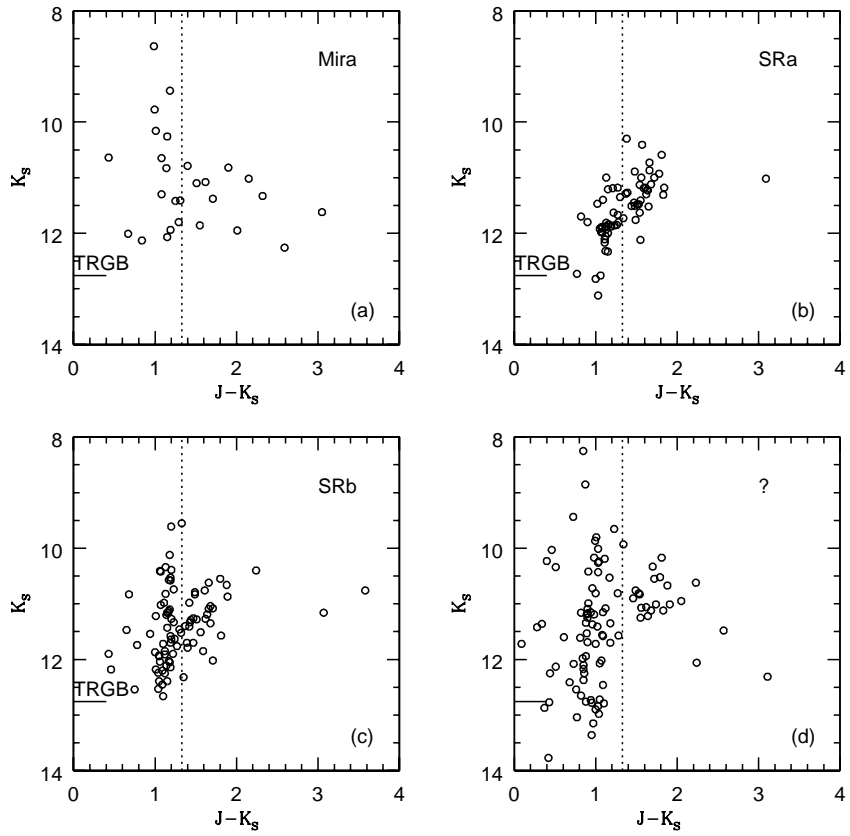

Fig. 5. Colour-magnitude diagrams $\left(J-K_{S}, K_{S}\right)$ for variable stars classified as Mira a), SRa b) and SRb c) according to their periodicity and amplitude of variation. d) is the same diagram for variable sources which could not be classified in one of the three categories because of low $S / N$ or an extremely irregular light-curve. The vertical dotted line indicates the separation between $\mathrm{O}-$ rich and $\mathrm{C}$-rich stars as in Fig. 3.

\section{Conclusions}

The stars selected from the ISO-MCMS with a confident DENIS/2MASS counterpart have been cross-identified with the MACHO database. The extracted light-curves have been analysed to derive the main period and amplitude of pulsation. The bolometric magnitude has been calculated by integrating the spectral energy distribution from the $I$ DENIS to the $L W 10$ ISO wave band. This, in combination with the correlation of Alard et al. (2001) between $M_{\mathrm{BOL}}$ and the mass-loss rate, indicates that most of the stars in the present sample lose $10^{-6} M_{\odot} \mathrm{yr}^{-1}$ of matter. A distinction between Mira and SR is obtained by applying an amplitude and regularity selection criterion. The histogram of the amplitude of pulsation of $\mathrm{O}$-rich and $\mathrm{C}$-rich stars has a similar distribution, but on average $\mathrm{C}-$ rich stars have a larger amplitude. This may indicate that either most of the C-rich stars in the SMC are of Mira type, or that the metallicity affects the amplitude in such a way that in a lower metallicity environment the amplitude of pulsation is larger. This effect cannot yet be checked for in other metalpoor galaxies in the Local Group, because despite the fact that many AGB stars have been discovered there is not enough information on their variability and type.

The most obscured stars have large $\left(K_{S}-L W 2\right)$ and $(L W 2-L W 10)$ colours. This indicates sources with a thicker circumstellar envelope and allows to easily distinguish AGB stars from supergiant stars and other classes of objects such as PN and emission line sources. The difference between the 2MASS and DCMC magnitudes suggests that most of the sources in the sample are variables.
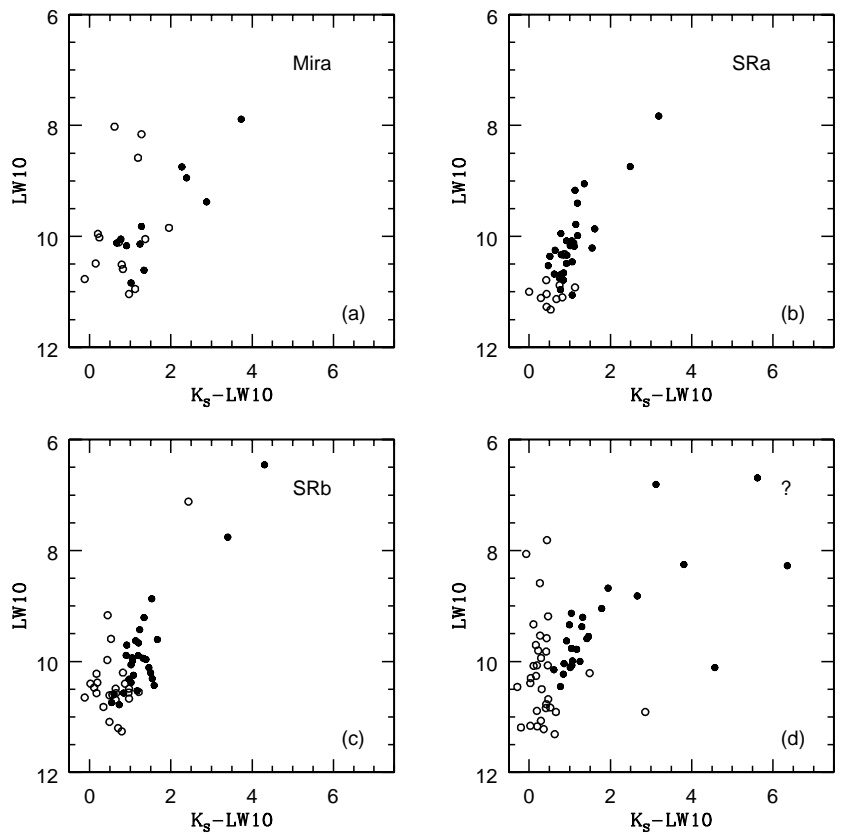

Fig. 6. Colour-magnitude diagrams $\left(K_{S}-L W 10, L W 10\right)$ for the same groups of sources as in Fig. 5.
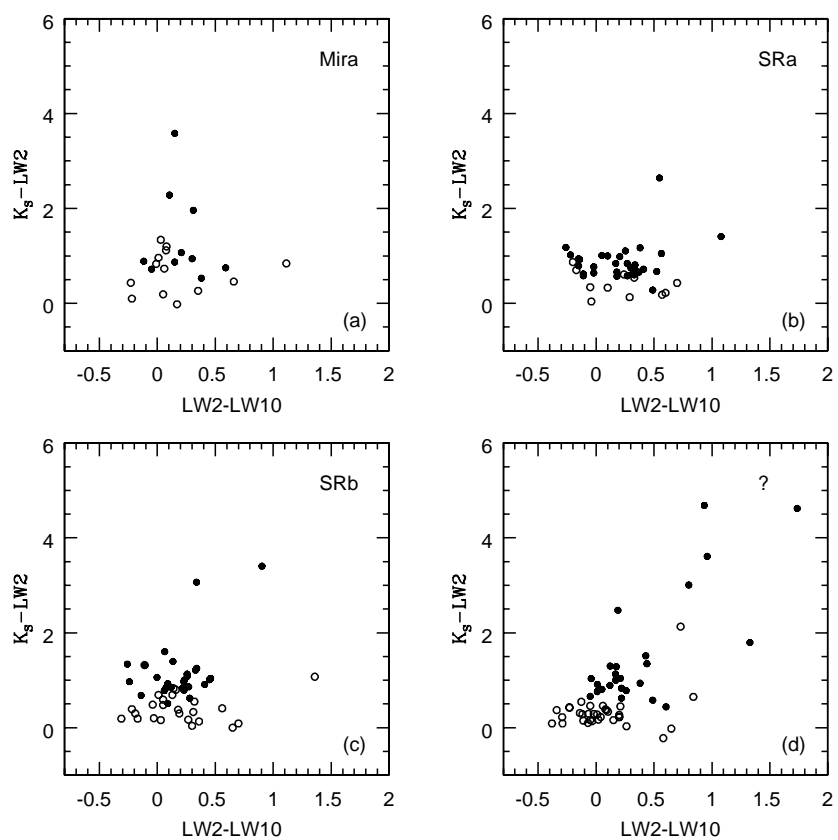

Fig. 7. Colour-colour diagrams $\left(K_{S}-L W 2, L W 2-L W 10\right)$ for the same groups of sources as in Fig. 5.

Acknowledgements. The first author is very grateful to J.-P. Beaulieu for providing the algorithm to detect the periodicities. This paper utilises public domain data obtained by the MACHO Project, jointly funded by the US Department of Energy through the University of California, Lawrence Livermore National Laboratory under contract No. W-7405-Eng-48, by the National Science Foundation through the Center for Particle Astrophysics of the University of California under cooperative agreement AST-8809616, and by the Mount Stromlo and Siding Spring Observatory, part of the Australian National University. This publication makes use of data products from the Two Micron All Sky Survey, which is a joint project of the University of Massachusetts 


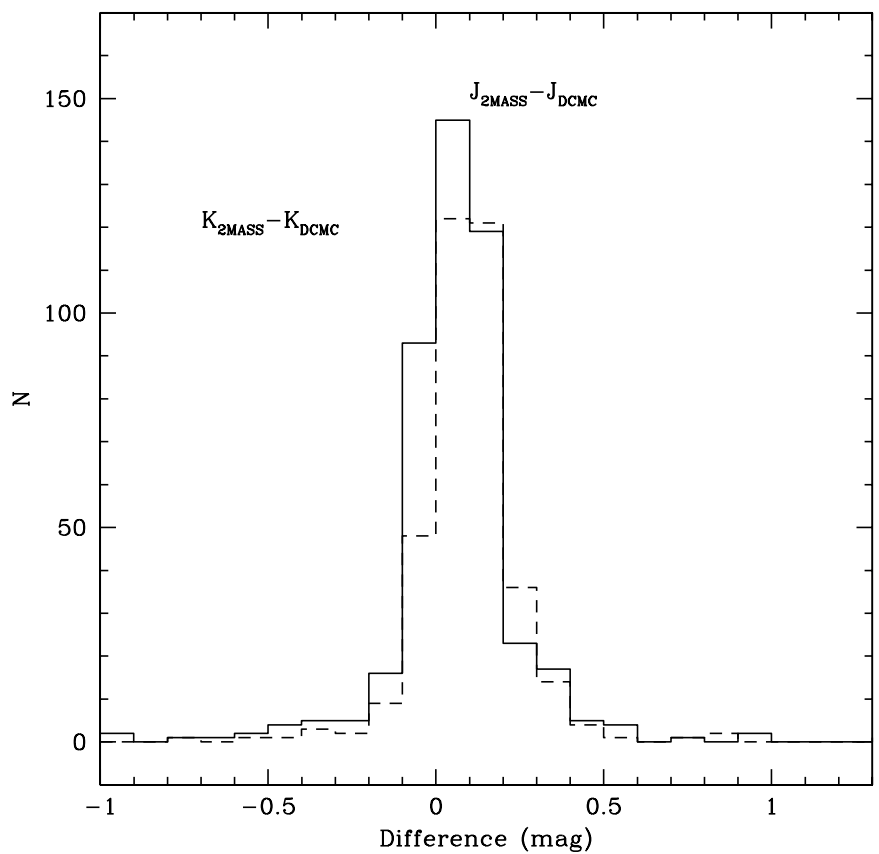

Fig. 8. Histogram of the differences between the 2MASS and DCMC magnitudes of all the sources in the sample.

and the Infrared Processing and Analysis center/California Institute of Technology, funded by the National Aeronautics and Space Administration and the Mational Science Foundation.

\section{Appendix A: The period-luminosity relation of Mira stars}

In order to improve our understanding of the identification of Mira-type variables we decided to re-analyse the LPVs studied by Feast et al. (1989). These are the Mira stars that define the period-luminosity relation in the LMC. We could find the B1950 coordinates of 42 out of the 55 sources with a "good" or "fair" light-curve as classified by Feast et al. (1989). We could not find in the literature the coordinates of the objects from the sample of Glass \& Lloyd Evans (1981) and for three objects from the list of Glass \& Reid (1985) or Reid et al. (1988). However, there still remain enough sources for our purpose.

We searched in the MACHO database for the light-curve of the closest LPV to these 42 sources. The identification was easy in most of the cases because of the highly regular and large amplitude light-curve typical of a Mira star. Five sources (W158, GR28, GR4, RGC91, RGC89) 3 are unfortunately outside the fields observed by MACHO. For one source the MACHO web site gives a persistent initialization error (W94) and for four additional sources (GR13, GR21, RGC37, RGC53) we could not find a nearby counterpart (a first possible candidate is further than $8^{\prime \prime}$ ). Only one source (W30) has two possible MACHO counterparts and due to the low accuracy of the initial coordinates compared to the MACHO coordinates we decided to

3 The designation of the sources follows that of Feast et al. (1989): $\mathrm{W}=$ Wood et al. (1985), GR = Glass \& Reid (1985) and we introduce RGC $=$ Reid et al. (1988). remove this source from the discussion. Finally we could successfully extract the light-curves of 31 sources.

We determined the main periodicity, as described in Sect. 3, of the sources with a MACHO-red light-curve. We obtained periods that are at most 20 days different from those derived by Feast et al. (1989) except for 4 sources which result to have a period coincident with one of the extremes of the searching window (GR2, GR3, GR18 and GR29, they have been removed from the following analysis) and source GR17 for which we derive a period of 51 days shorter. The period of source RGC20 falls in the region of aliases (Sect. 3), thus the source has been removed from the following discussion. The quality parameter of the 26 sources with a convincing period determination is always above 100 and the difference between the assigned period and the mean of all the determinations with $q>15$ is of the order of 1 day for most of the sources but always below 10 days. These numbers may be different in the case of MACHO-blue light-curves.

Table A.1 lists the name of the sources (Col. 1), the coordinates at the epoch $\mathrm{J} 2000$ precessed from the original coordinates (Cols. 2 and 3), the MACHO identification code (Cols. 46), the distance in arcsec between the Feast et al. (1989) source and the MACHO counterpart (Col. 7), the period, amplitude and the value of the quality parameter for the red light-curve (Cols. 8-10).

We conclude that by selecting, from our sample, large amplitude sources with a single periodicity and $q>100$ we are identifying Mira variables of the same type as those studied by Feast et al. (1989). They define a tight PLR and the small scatter in Fig. 3d is probably due to the availability of only one epoch bolometric magnitude. Note that with this selection criterion we also find sources at very bright magnitudes $\left(M_{\mathrm{BOL}}=-7\right)$ which deviate from the extrapolation of the PLR defined only for sources with $P<420$ days. We estimate from Fig. $3 \mathrm{~b}$ that these stars have $M>5 M_{\odot}$ and as previously noted by Feast et al. (1989) the PLR breaks at high masses. These stars are likely to be "over-luminous" as a result of Hot Bottom Burning (Zijlstra et al. 1996; Whitelock et al. 2003). However, because of their very high luminosity they could also be supergiants.

\section{Appendix B: Bolometric correction(s)}

\section{B.1. $B C$ l versus $I-J$}

Figure B.1 compares the bolometric correction obtained from the integration under the SED (Sect. 3.1) and the relation derived by Alvarez et al. (2000) for cool O-rich stars. The agreement is very good for the O-rich stars of our sample. This confirms that the relation is valid for the SMC as well as for the LMC and the Bulge. However this relation overestimates the correction for $\mathrm{C}$-rich variables in the SMC. On the other hand we should check that the integration under the SED does not underestimates the bolometric correction (if the stars have a circumstellar envelope and dust extinction the correction for energy re-radiated by dust becomes important). Our measurements have been corrected for interstellar extinction while the relation has not. The agreement for $\mathrm{O}$-rich stars might be 
Table A.1. Table of MACHO counterparts of Feast et al. (1989) sources.

\begin{tabular}{|c|c|c|c|c|c|c|c|c|c|c|}
\hline Source & $\mathrm{Ra}$ & 2000 & Tile & Seq. & No. & Dis. & $m_{\mathrm{BOL}}$ & $P$ & $\begin{array}{c}A \\
\text { RED }\end{array}$ & $q$ \\
\hline W132 & $5: 28: 54.13$ & $-69: 40: 15.33$ & 77 & 7914 & 16 & 2.71 & 14.48 & 158 & 219 & 594 \\
\hline W151 & $5: 29: 28.84$ & $-69: 32: 53.79$ & 77 & 7916 & 24 & 1.74 & 14.67 & 173 & 292 & 667 \\
\hline W148 & $5: 29: 25.19$ & $-69: 26: 54.50$ & 77 & 7917 & 61 & 2.73 & 14.74 & 183 & 417 & 216 \\
\hline W19 & $5: 26: 23.89$ & $-69: 34: 00.44$ & 77 & 7431 & 18 & 4.35 & 14.33 & 196 & 119 & 172 \\
\hline W77 & $5: 28: 6.25$ & $-69: 32: 28.82$ & 77 & 7795 & 15 & 2.33 & 14.20 & 209 & 166 & 656 \\
\hline W74 & $5: 27: 51.13$ & $-69: 58: 09.88$ & 77 & 7667 & 943 & 3.16 & 14.53 & 233 & 392 & 791 \\
\hline W1 & $5: 25: 49.46$ & $-69: 44: 36.01$ & 77 & 7429 & 303 & 3.37 & 14.55 & 237 & 508 & 1142 \\
\hline W140 & $5: 29: 16.66$ & $-69: 43: 34.97$ & 77 & 7913 & 369 & 1.93 & 14.17 & 243 & 365 & 1155 \\
\hline W48 & $5: 27: 10.38$ & $-69: 28: 27.76$ & 77 & 7554 & 11 & 2.94 & 13.97 & 280 & 453 & 1251 \\
\hline W46 & $5: 27: 09.34$ & $-69: 41: 57.76$ & 77 & 7550 & 22 & 2.50 & 14.20 & 297 & 107 & 274 \\
\hline W126 & $5: 28: 41.48$ & $-69: 51: 15.48$ & 77 & 7790 & 310 & 3.22 & 13.91 & 315 & 612 & 1114 \\
\hline W103 & $5: 28: 27.36$ & $-69: 49: 03.44$ & 77 & 7791 & 104 & 2.65 & 14.14 & 366 & 368 & 419 \\
\hline GR5 & $5: 12: 38.39$ & $-65: 56: 10.10$ & 57 & 5308 & 13 & 2.85 & 15.05 & 140 & 394 & 176 \\
\hline RGC60 & $5: 26: 34.72$ & $-67: 51: 58.68$ & 4 & 7578 & 16 & 1.23 & 14.64 & 157 & 261 & 141 \\
\hline GR27 & $5: 29: 01.37$ & $-65: 29: 34.64$ & 65 & 7977 & 11 & 0.63 & 14.31 & 194 & 241 & 108 \\
\hline GR7 & $5: 07: 08.26$ & $-66: 35: 23.75$ & 56 & 4330 & 282 & 1.97 & 14.51 & 208 & 484 & 190 \\
\hline RGC55 & $5: 29: 12.42$ & $-67: 57: 30.10$ & 4 & 7940 & 12 & 2.42 & 14.25 & 283 & 170 & 156 \\
\hline GR10 & $5: 05: 02.81$ & $-66: 53: 10.92$ & 53 & 4084 & 14 & 0.88 & 13.73 & 306 & 323 & 295 \\
\hline GR26 & $5: 34: 15.15$ & $-65: 29: 49.35$ & 65 & 8823 & 24 & 1.01 & 14.28 & 306 & 248 & 131 \\
\hline GR30 & $5: 24: 40.13$ & $-65: 41: 18.83$ & 63 & 7248 & 11 & 1.30 & 13.71 & 315 & 419 & 264 \\
\hline W220 & $5: 30: 43.00$ & $-70: 02: 39.33$ & 77 & 8150 & 42 & 5.61 & 14.12 & 283 & 177 & 1121 \\
\hline GR11 & $5: 02: 52.87$ & $-67: 07: 40.78$ & 25 & 3717 & 1133 & 0.06 & 13.92 & 322 & 184 & 282 \\
\hline GR12 & $5: 03: 49.17$ & $-66: 15: 57.55$ & 55 & 3851 & 16 & 1.52 & 12.36 & 592 & 463 & 329 \\
\hline GR1 & $5: 15: 40.39$ & $-66: 04: 59.15$ & 59 & 5790 & 13 & 2.74 & 12.24 & 535 & 543 & 260 \\
\hline GR48 & $5: 23: 53.00$ & $-66: 41: 29.69$ & 60 & 7112 & 15 & 1.67 & 11.87 & 634 & 505 & 217 \\
\hline GR17 & $5: 39: 32.97$ & $-66: 56: 35.78$ & 67 & 9649 & 46 & 2.24 & 11.87 & 729 & 532 & 384 \\
\hline
\end{tabular}

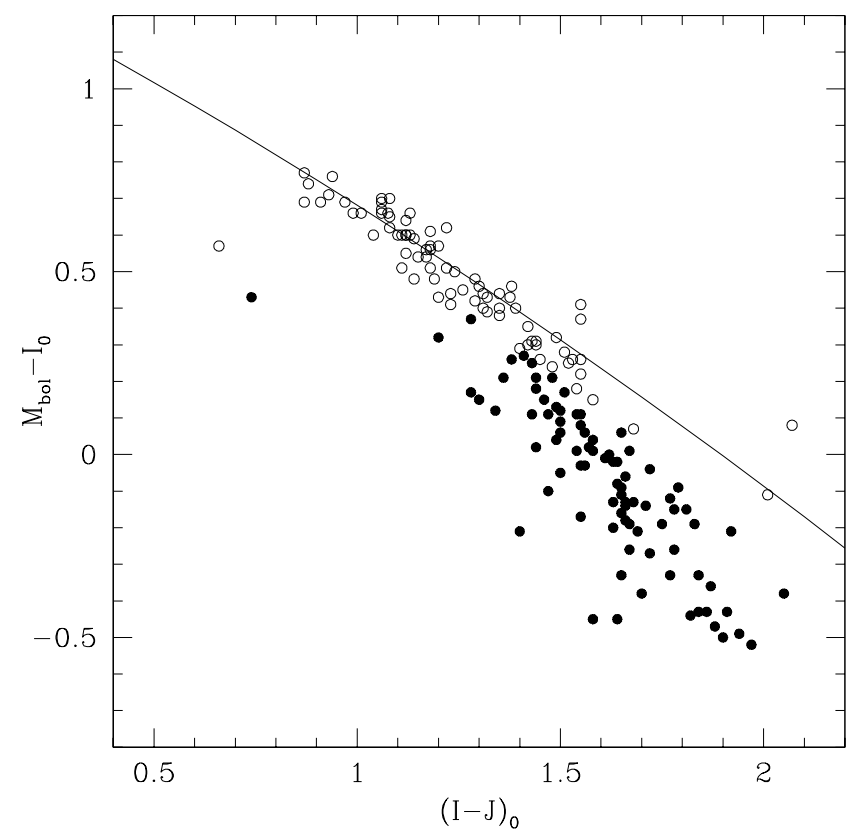

Fig. B.1. The bolometric correction $\mathrm{BC}_{I}$ as a function of the $I-J$ colour for the $\mathrm{O}-$ rich (open circles) and $\mathrm{C}$-rich (filled circles) variable stars in the sample. The continuous line is the relation derived by Alvarez et al. (2000) for cool O-rich stars. a consequence of the systematic difference between the DENIS filters, used in this work, and the broad pass-bands by Bessels \& Brett (1988) and Bessel (1990) used to derive the relation. The disagreement for C-rich stars might be a consequence of the $\mathrm{SiC}$ dust feature which causes the spline not to follow the continuum of the SED. However the data of Alvarez et al. (2000) include only 6 C-rich stars and a total of four stars belonging to the LMC or the SMC. In addition the spectral range covered by their measurements is limited to $2.5 \mu \mathrm{m}$. The zeropoint of the bolometric scale is the same in both studies.

\section{B.2. $B C_{K}$ versus $J-K$}

Figure B. 2 compares the bolometric correction obtained as in Sect. 3.1 with the relation derived by Montegriffo et al. (1998) for Population II giants of low $([\mathrm{Fe} / \mathrm{H}]<-1.0)$ and "high" metallicity $([\mathrm{Fe} / \mathrm{H}]>-1.0)$. They used $M_{\mathrm{BOL}, \odot}=4.75$. Their relation was defined up to $(J-K)=0.8$ but being the metalpoor relation very close to the metal-rich relation at redder colours we consider the latter as a possible extrapolation. This results in a fairly good fit for the $\mathrm{O}$-rich variables while $\mathrm{C}$-rich variables lie systematically below the relation. This is a similar effect as that observed for $B C_{I}$ versus $I-J$ (Fig. B.1). The giants in the sample of Montegriffo et al. (1998) are 


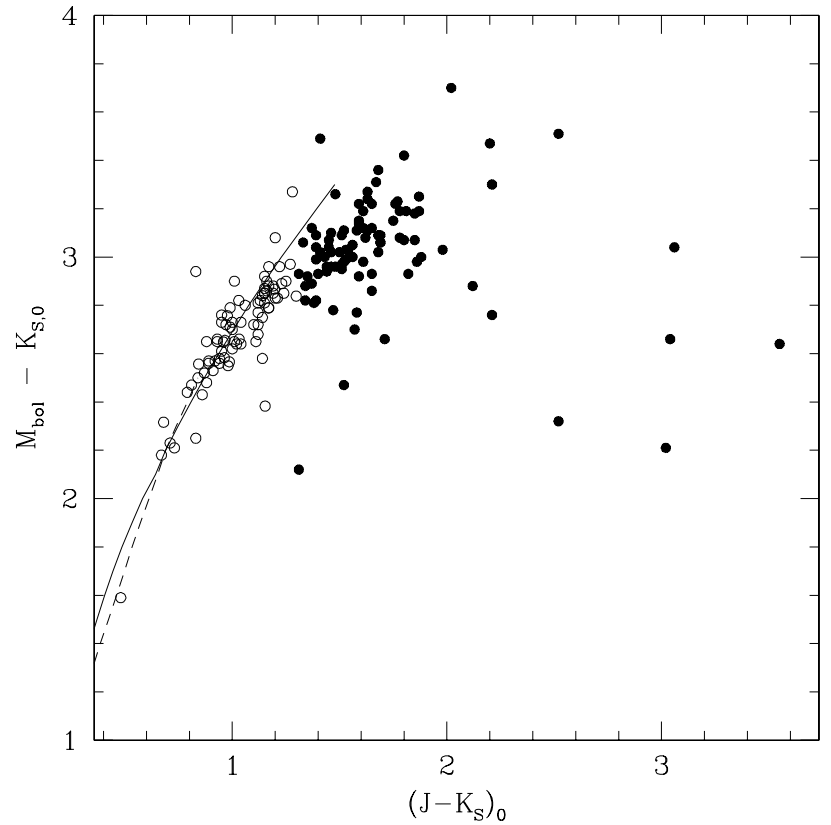

Fig. B.2. The bolometric correction $B C_{K}$ as a function of the $J-K_{S}$ colour for the O-rich (empty circles) and C-rich (filled circles) variable stars in the sample. The lines are the relations derived by Montegriffo et al. (1998) for Population II giants of low (dashed line) and high metallicity (continuous line).

members of galactic globular clusters. C-rich stars are rare members of these systems. The spectral range covered by the observations is limited to $3.5 \mu \mathrm{m}$ ( $L$ band) and they derive the bolometric magnitude by integrating the SED which is previously re-constructed by means of a set of Plank functions at a given wavelength. These effects may influence the correction to be applied to the flux measured only in the $K$ band. Besides the $2.2 \mu \mathrm{m}$ filters used in both studies are not strictly the same. We conclude that we cannot at present compare the correction for C-rich stars while $\mathrm{O}$-rich stars are qualitatively in agreement. It would be useful to compare stars of other galaxies in the Local Group at a different metallicity.

\section{References}

Alard, C., Blommaert, J., Cesarsky, C., et al. 2000, ApJ, 552, 289

Alcock, C., Allsman, R. A., Alves, D. R., et al. 2000, ApJ, 542, 281

Alvarez, R., Lançon, A., Plez, B., \& Wood, P. R. 2000, A\&A, 353, 322

Bessel, M. S. 1990, A\&A, 83, 357

Bessel, M. S., \& Brett, J. M. 1988, PASP, 100, 1143

Cioni, M.-R. L., \& Habing, H. J. 2003, A\&A, 402, 133

Cioni, M.-R. L. 2002a, Workshop on Mass-Losing Pulsating Stars and their Circumstellar Matter, ed. Y. Nakada, \& M. Honma, Kluwer ASSL serie [astro-ph/0207619]

Cioni, M.-R. L., Marquette, J.-B., Loup, C., et al. 2001, A\&A, 377, 945

Cioni, M.-R. L., van der Marel, R. P., Loup, C., \& Habing, H. J. 2000, A\&A, 359, 601
Cioni, M.-R., Loup, C., Habing, H. J., et al. 2000a, A\&AS, 144, 235

Delmotte, N., Loup, C., Egret, D., et al. 2002, A\&A, 396, 143

Egan, M. P., Van Dyk, S. D., \& Price, S. D. 2001, ApJ, 122, 1844

Epchtein, N., de Batz, B., Capoani, L., et al. 1997, The Messenger, 87, 27

Feast, M. W., Glass, I. S., Whitelock, P. A., \& Catchpole, R. M. 1989, MNRAS, 241, 375

Gardiner, M. T., \& Noguchi, M. 1996, MNRAS, 278, 191

Glass, I. S. 1999, in Handbook of Infrared Astronomy (Cambridge University Press)

Glass, I. S., \& Lloyd Evans, T. 1981, Nature, 291, 303

Glass, I. S., \& Reid, N. 1985, MNRAS, 214, 405

Glass, I. S., \& Schultheis, M. 2002, MNRAS, accepted [astro-ph/0208253]

Heller, P., \& Rohlfs, K. 1994, A\&A, 291, 743

Kiss, L. L., Szatmáry Szabó, G., \& Mattei, J. A. 2000, A\&AS, 145, 283

Kuĉinska, A., Lindegren, L., Tanabé, T., \& Vanseviĉius, V. 2002, [astro-ph/0212185]

Lasserre, T., Alfonso, C., Albert, J. N., et al. 2000, A\&A, 355, L39

Lebzelter, T., Schultheis, M., \& Melchior, A. L. 2002, A\&A, 393, 573

Lloyd Evans, T., Glass, I. S., \& Catchpole, R. M. 1988, MNRAS, 231, 773

Loup, C., Cioni, M.-R. L., Habing, H. J., et al. 2002, submitted

Menzies, J., Feast, M., Toshihiko, T., et al. 2002, MNRAS, accepted [astro-ph/020529]

Montegriffo, P., Ferraro, F. R., Origlia, L., \& Fusi Pecci, F. 1998, MNRAS, 297, 872

Moore, B., \& Davis, M. 1994, MNRAS, 270, 209

Noda, S., Takeuti, M., Abe, F., et al. 2002, MNRAS, 330, 137

Omont, A., Ganesh, S., Alard, C., et al. 1999, A\&A, 348, 755

Percy, J. R., \& Bagby, D. H. 1999, PASP, 111, 203

Price, S. D., Egan, M. P., Carey, S. J., et al. 2001, AJ, 121, 2819

Rebeirot, E., Martin, N., Mianes, P., et al. 1983, A\&AS, 51, 277

Reid, N., Glass, I. S., \& Catchpole, R. M. 1988, MNRAS, 232, 53

Rejkuba, M. 2002, Ph.D. Thesis, Universidad Católica de Chile

Schultheis, M., Ganesh, S., Glass, I. S., et al. 2000, A\&A, 362, 215

Schwarzenberg-Czerny, A. 1996, ApJ, 460, L107

van Loon, J. Th., Zijlstra, A. A., Whitelock, P. A., et al. 1998, A\&A, 329,169

van Loon, J. Th. 2000, A\&A, 354, 125

van Loon, J. Th., Zijlstra, A. A., Bujarrabal, V., \& Nyman L. A. 2001, A\&A, 368, 950

Vassiliadis, E., \& Wood, P. R. 1993, ApJ, 413, 641

Westerlund, B. E. 1997, in The Magellanic Clouds, Cambridge Astrophysics Series, 29, 6

Whitelock, P. A., Feast, M., Marang, F., \& Overbeek, M. D. 1997, MNRAS, 288, 512

Whitelock, P. A., Feast, M. W., van Loon, J. Th., \& Zijlstra, A. A. 2003, MNRAS, accepted [astro-ph/0302246]

Winters, J. M., Fleischer, A. J., Gauger, A., et al. 1994, A\&A, 290, 623

Wood, P. R., Bessel, M. S., \& Paltoglou, G. 1985, AJ, 290, 477

Wood, P. R., Whiteloak, J. B., Hughes, S. M. G., et al. 1992, ApJ, 397 , 552

Wood, P. R., Alcock, C., Allsman, R. A., et al. 1999, IAU Symp., 191, 151

Zebruń, K., Soszyński, I., Woźniak, et al. 2002, Acta Astron., 51, 317

Zijlstra, A. A., Loup, C., Waters, L. B. F. M., et al. 1996, A\&A, 279, 32 